\title{
Lattice relaxations and hyperfine fields of heavy impurities in $\mathrm{Fe}$
}

\author{
T. Korhonen \\ Laboratory of Physics, Helsinki University of Technology, P.O. Box 1100, FIN-02015 HUT, Finland
}

\author{
A. Settels, N. Papanikolaou, R. Zeller, and P. H. Dederichs \\ Institut für Festkörperforschung, Forschungszentrum Jülich, D-52425 Jülich, Germany
}

(Received 22 December 1999)

\begin{abstract}
We present first-principles calculations of the lattice relaxations and hyperfine fields of heavy impurities in bcc Fe. We consider impurities of the $5 s p$ and $6 s p$ series, containing the largest atoms in the periodic table. As an application we calculate the hyperfine fields of these impurities and in particular the effects of lattice relaxations on these fields. The calculations are based on a full-potential Korringa-Kohn-Rostoker Green'sfunction method for defects and employ the local spin-density approximation for the exchange and correlation effects. The nonspherical parts of the potential and the charge density are included in the calculations and the forces are calculated by an ionic version of the Hellmann-Feynman theorem. The resulting lattice relaxations are relatively small, even for the largest impurities considered. The comparison of the calculated hyperfine fields with the experimental data shows that the inclusion of lattice relaxations improves the overall agreement with experiment.
\end{abstract}

\section{INTRODUCTION}

A point defect in a crystal, such as a vacancy or an impurity atom, presents not only a potential inhomogeneity, but also induces displacements of the neighboring host atoms from their ideal lattice positions. These lattice relaxations are, in fact, long ranged and lead to a volume change of the crystal. Very complete information about this displacement field can be obtained by diffuse x-ray/neutron-scattering experiments, but unfortunately very few systems have been measured. Detailed information on many more systems has been obtained by extended x-ray-absorption fine-structure (EXAFS) measurements, yielding reliable data for the nearest-neighbor shifts. In addition, lattice parameter measurements are available for many systems, giving direct information about the volume changes induced by the impurities. Many other defect properties, like solution energies or interaction properties, residual resistivities, and hyperfine interaction properties are influenced by these lattice relaxations and a detailed understanding of these properties is still far from complete.

The theoretical treatment of structural relaxations due to defects is a difficult task, in particular for transition-metal systems. In the past this problem has been mostly dealt with on a phenomenological basis, e.g., by applying models of lattice statics or by molecular dynamics with empirical potentials. Ab initio calculations have been restricted to supercell calculations or calculations for finite clusters. ${ }^{1}$ Recently we performed systematic ab initio calculations for transitionmetal impurities in copper ${ }^{2}$ and aluminum ${ }^{3}$ based on the Korringa-Kohn-Rostoker (KKR) Green's-function method and obtained a very good agreement with EXAFS (Ref. 4) and lattice parameter ${ }^{5,6}$ measurements. Typically the obtained relaxations in these systems are not very large, e.g., a $\mathrm{Zr}$ impurity in $\mathrm{Cu}$, which as an atom is twice as big as $\mathrm{Cu}$, shifts the nearest $\mathrm{Cu}$ neighbors by only $3.5 \%$ of the nearestneighbor (NN) distance. ${ }^{2}$ This finding is in line with the gen- eral notion that for steric reasons relaxations in close-packed metals are very small, considerably smaller than, e.g., for defects in semiconductors where open structures like diamond or zinc blende prevail. In order to learn more about the size of the relaxations in metals, in particular, for very large impurity atoms, we present in this paper KKR calculations for impurities of the $5 s p$ and $6 s p$ series in bcc Fe. To these series belong atoms which have the largest atomic volumes of all elements in the periodic table. For instance, the noble gas atoms $\mathrm{Xe}$ and $\mathrm{Rn}$ have atomic volumes of 37 and $50.5 \AA^{3}$, respectively, while the alkali metal atoms Cs $\left(71 \AA^{3}\right)$ and Fr, the largest element with a volume of $84 \AA^{3}$, are even larger, in particular, much larger than $\mathrm{Fe}$ with an atomic volume of $7.1 \AA^{3}$. For these reasons also particularly large lattice relaxations are to be expected. All these heavy $s p$ elements are characterized by very large positive solution enthalpies ${ }^{7}$ in $\mathrm{Fe}$ and thus they have a nearly vanishing solubility so that they can only be introduced into Fe by ion implantation at low temperatures.

The present calculations are motivated by recent measurements ${ }^{8,9}$ of the hyperfine fields of Cs and in particular of $\mathrm{Fr}$ in $\mathrm{Fe}$, which were performed by the nuclear orientation (NO) method and by nuclear magnetic resonance on oriented nuclei (NMR/ON) on Fe samples prepared by ion implantation at low temperatures. The measured hyperfine field of Fr is a factor of 5 smaller than the previous KKR calculations ${ }^{10-12}$ predicted, which were, however, based on spherical potentials, i.e., using atomic sphere approximation (ASA), and neglected lattice relaxations. It is natural to assume that the large relaxations induced by the Fr atom are the reason for this discrepancy. Thus, in addition to calculate the lattice relaxations of these king-size atoms, the present paper aims at studying the effects of lattice relaxations on the hyperfine fields of these impurities in Fe.

The hyperfine fields of impurities in the ferromagnetic hosts $\mathrm{Fe}, \mathrm{Co}$, and $\mathrm{Ni}$ have been extensively studied and a huge amount of experimental data exists. ${ }^{13,14}$ Only data for a few exotic impurities are missing, and in the case of $\mathrm{Fe}$, 
some have been added recently. ${ }^{8,9}$ Along each $s p$ series the impurity hyperfine fields show a very systematic trend: they are negative at the beginning of each series, rise to large positive values at the end of series, and drop sharply back to negative values at the beginning of the next row. This systematic trend was explained by the Kanamori group. ${ }^{15-17}$ These authors noted that the trend arises from the hybridization of the impurity $s$ and the host $d$ states into bonding $s d$ hybrids, which show a preferential occupation of the minority states, and into spin-split antibonding hybrids. At the beginning of each series only the bonding hybrids are occupied yielding negative fields. Later on also the spin-split antibonding peaks are occupied. Here the occupation of the lowerlying antibonding peaks in the majority bands leads to a strong increase of the hyperfine fields with large positive values at the end of the series, to be followed by a strong drop to negative values once the minority antibonding states are filled for the impurities with higher valency. The exact position of the maximum hyperfine field depends on the bonding-antibonding splitting and thus on the strength of the hybridization between the impurity $s$ and Fe $d$ states. Since the hybridization should be strongly modified by lattice relaxations, it is not too surprising that the KKR-ASA calculations, which did not consider displacement effects, led to unrealistically large values for the hyperfine fields of Cs and Fr.

For the reasons above we calculated the lattice relaxations and the resulting effects on the hyperfine fields for heavy impurities of the $5 s p$ and $6 s p$ series in Fe using a fullpotential KKR Green's-function method. The organization of the paper is as follows. In Sec. II, the computational method is presented first for the bulk (Sec. II A) and then for the impurity (Sec. II B) problem. In Sec. III, the calculation of impurity induced lattice relaxations around $5 s p$ and $6 s p$ impurities in bcc Fe are presented and discussed. Section IV contains the results obtained for the hyperfine fields and the related discussion. Section V is a short summary.

\section{THEORETICAL METHOD}

Our calculations are based on the density-functional theory with the exchange and correlation effects being treated in the local spin-density approximation (LSDA), for which the parametrization of Vosko, Wilk, and Nusair ${ }^{18}$ to the Monte Carlo data of Ceperley and Alder ${ }^{19}$ is used. The calculations are performed using a full-potential KKR Green's-function method for defects which is an all-electron method and treats also the nonspherical parts of the potential and the charge density correctly. The method takes the advantage of a Green's-function method to embed a cluster containing the impurity atom and a few disturbed neighboring host shells correctly in the ideal crystal, which is ideal for the case now studied, i.e., iron alloys in the dilute limit. Lattice statics methods are used to describe the longer ranged relaxations and to speed up the determination of the final positions of the atoms from the $a b$ initio forces, which are calculated using an ionic version of the Hellmann-Feynman theorem. ${ }^{2}$ This method was shown to give a realistic description of lattice relaxations around substitutional impurities in metals. ${ }^{2,3}$

The calculations are done using the scalar relativistic ap- proximation, i.e., the spin-orbit coupling is neglected, and the hyperfine fields are calculated both at the ideal and the fully relaxed positions in order to study the effect of lattice relaxations on the fields. The theoretical LSDA lattice constant and the calculated Born-von Karman coupling parameters of $\mathrm{Fe}$ are used in the calculations. An angular momentum cutoff $l_{\max }=4$ is used for the Green's function and for the radial functions, which implies a cutoff $2 l_{\max }$ for the expansion of the potentials and charge density and a cutoff $4 l_{\max }$ for the shapes of the Wigner-Seits (WS) cells. The space is devided into nonoverlapping WS cells, which are further divided into muffin-tin (MT) spheres and to the interstitial space outside the MT spheres and the "nonspherical" coupled radial equations are solved by using the second Born approximation for the nonspherical part of the potential. ${ }^{20} \mathrm{~A}$ logarithmic radial mesh is used inside the MT spheres and an equally spaced radial mesh is used outside the spheres. The method proposed in Ref. 21 to cope with the discontinuities arising from the sharp edges of the WS cells is employed.

\section{A. Bulk calculation}

In our multiple-scattering KKR Green's-function method the starting point is the free electron Green's function which is analytically known. The Green's function of the host crystal, $G^{0}\left(\mathbf{r}, \mathbf{r}^{\prime}, E\right)$, is obtained by a $\mathbf{k}$-space integration. After this the electron density $n(\mathbf{r})$ is obtained from the imaginary parts of the site-diagonal elements of the Green's function by an energy integration

$$
n(\mathbf{r})=-\frac{1}{\pi} \int_{E_{B}}^{E_{F}} \operatorname{Im}\left[G^{0}(\mathbf{r}, \mathbf{r}, E)\right] d E .
$$

Here the energy integral extends over all occupied valence states from a suitably chosen energy $E_{B}$ between the valence band and the core states up to the Fermi energy $E_{F}$. By using analytical properties of the Green's function the energy integration in Eq. (1) can be replaced by a contour integral in the (upper) complex energy plane. This contour integral can be evaluated with much fewer energy points, because the sharp structures of the Green's function and the density of states (DOS) are smoothening out quite rapidly when going away from the real axis. In this work a recent extension to finite temperatures, ${ }^{22}$ based on a Fermi-Dirac distribution for the occupation function, is used. A half-rectangle-shaped contour starting at $E_{B}$ and an electronic temperature of $T$ $=800 \mathrm{~K}$ with five Matsubara points are used, so the horizontal line extending to the infinity lies $10 \pi k T(\approx 160 \mathrm{mRy})$ above the real axis. In total 30 energy points are needed for the calculation of the valence states. The accuracy of the contour integration was tested by doubling the number of energy points and it was found to be better than $10^{-7}$ electrons for the partial $s, p, d, \ldots$ charges.

During the lattice constant minimization the MT sphere radius was kept constant. A radius of 1.8027 a.u. together with a semicore treatment described below was used. The same MT radius should be used for different lattice constant values in order to avoid inaccuracies in the core calculation due to slightly different radial meshes and due to the fact that the core electrons are supposed to reside completely inside 
the MT sphere. The minimization gave a value of 5.204 a.u. for the lattice constant and the bulk modulus was found to be 2.6 Mbars.

In our treatment the core electrons are assumed to reside completely inside the nonoverlapping MT spheres and they are calculated using spherically averaged potentials. The uppermost core states of iron, $3 s$ and $3 p$, fit just about inside a MT sphere touching the WS cell boundaries, but, e.g., for early $3 d$ metals these states extend over the MT radius. The same situation occurs for the uppermost core states of the now studied heavy impurities in Fe and also for the Fe $3 s$ and $3 p$ core states when one is using a relatively small MT radius, which is needed when lattice relaxations are included. So, these so-called "semicore" states should be included in the energy contour thereby treating them as valence states. Papanikolaou et $a l^{2}$ showed that for the early $3 d$ and, in particular, for the early $4 d$ impurities in $\mathrm{Cu}$ the inclusion of the impurity semicore states in the energy contour is needed in order to get good forces (the $\mathrm{Cu} 3 s$ and $3 p$ electrons are sufficiently localized not to cause any troubles). For the present case, one cannot even converge the bulk Fe with the $3 s$ and $3 p$ treated as core states, when a $20 \%$ smaller than touching MT sphere demanded by the now calculated relatively large lattice relaxations is used. ${ }^{23}$

The inclusion of the semicore states in the energy contour is in principle straightforward within the KKR formalism, one just extends the energy contour below the uppermost core states. The semicore states are lying quite deep and are thus well localized and produce sharp peaks on the real energy axis. For an optimal integration of the semicore states a long rectangular contour with a large imaginary part $\operatorname{Im}(E)$ $=1.0 \mathrm{Ry}$ is used in this work. The semicore contour starts 6.2 Ry below the valence contour and it returns back to the real axis where the valence contour starts. With about 50 energy points for the semicore a similar accuracy as for the valence states is obtained.

In actual calculations the treatment of the semicore electrons of the host is not so straightforward since the charge density and the DOS in each cell are calculated by a summation over angular momenta up to a cutoff $l_{\max }$. Due to this cutoff the semicore charge shows slight deviations from the correct integer value, with a typical error of about $10^{-3}$ electrons. Due to the charge neutrality this error in the semicore charge is in the self-consistency iterations automatically balanced by an opposite contribution in the valence charge, so that the Fermi level will depend (weakly) on the semicore. In the total-energy calculation this fact leads to an error in the single-particle energies of a few $\mathrm{mRy}$ in the present case. Moreover this error systematically increases for smaller lattice constants due to the increasing overlap of the semicore orbitals. This is illustrated in Fig. 1, where the resulting totalenergy curve (unscaled semicore) yields a considerably smaller lattice constant than, e.g., a calculation, where the semicore electrons are treated as core electrons (no semicore). This problem does not occur in the real-space impurity calculations, because the Lloyd's formula ${ }^{24}$ is used for the evaluation of the single-particle energies and this includes an implicit summation over all angular momenta.

The above error can be corrected by using a renormalized semicore DOS, $\tilde{n}_{\mathrm{sc}}(E)$, in the energy calculation where the DOS, $n_{\mathrm{sc}}(E)$, is multiplied by a constant in order to obtain an

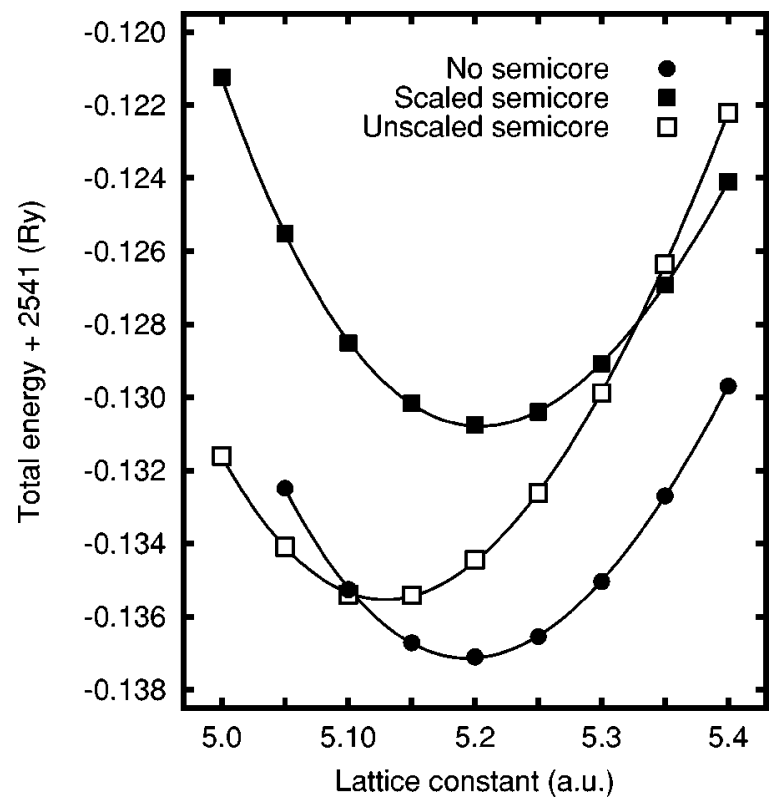

FIG. 1. Binding-energy curves for bcc Fe using different schemes to treat the semicore electrons (see text). The curve obtained using the energy correction (2) is not shown, because it practically coincides with the curve obtained by using scaled semicore.

integer semicore occupancy. The formula for the singleparticle energy, $E_{\mathrm{sc}}=\int_{\mathrm{sc}} E n_{\mathrm{sc}}(E) d E$, is then replaced by

$$
\widetilde{E}_{\mathrm{sc}}=\int_{\mathrm{sc}} E \tilde{n}_{\mathrm{sc}}(E) d E-E_{F} \Delta N_{\mathrm{sc}},
$$

where $\Delta N_{\mathrm{sc}}$ represents the missing (or surplus) semicore charge needed for integer occupancy. The weak dependence of the Fermi level, $E_{F}$, on the proper number of semicore electrons is taken care by the second term: $E_{F} \Delta N_{\mathrm{sc}}$ $\approx E_{F} n\left(E_{F}\right) \Delta E_{F}$.

Another possibility to correct the error is to use the renormalized semicore charge everywhere in the self-consistency iterations, so that integer charges are also used in the double counting terms. These additional corrections should be small, since due to the extremal properties of the total energy the double counting terms are extremal against small variations in the charge density. The result of this latter procedure (scaled semicore) is also illustrated in Fig. 1 and it is seen to yield practically the same lattice constant as the "full core", treatment (no semicore) does, where larger MT spheres were used to avoid the problems with semicore. The result using the renormalized semicore DOS without a renormalized charge density, i.e., using only the energy correction (2), is not shown since it practically coincides with the "double" renormalized result.

\section{B. Impurity calculation}

When the host potential and the host Green's function are known, the Green's function for the impurity problem can be obtained from a Dyson equation in real space, being essentially determined by the perturbation of the potential. Due to the efficient screening in metals, the size of the potential perturbation is naturally restricted. In this work the potentials of five neighboring shells of $\mathrm{Fe}$ atoms are allowed to be 


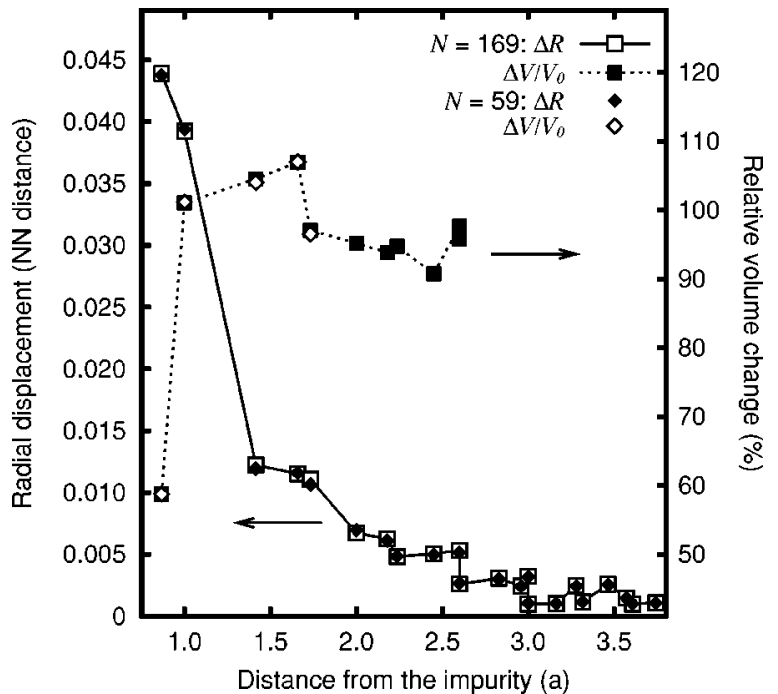

FIG. 2. Radial relaxation pattern around a Fr impurity calculated by the lattice static model starting from the $a b$ initio forces. Clusters with 59 and 169 perturbed potentials at ideal bcc lattice sites were used. The evolution of the impurity induced relative volume changes $\Delta V / V_{0}$ are also shown, when more and more $\mathrm{NN}$ shells are included in the sum (3).

perturbed, so that in total 59 perturbed potentials are recalculated self-consistently in the impurity iterations. Grouptheoretical methods based on the $O_{h}$ point group are used to reduce the computational effort. To check if the chosen cluster size was large enough, calculations for a Fr impurity were performed using a larger cluster with $N=169$ perturbed potentials, i.e., it contained the impurity and the first 11 neighboring shells of Fe. Figure 2 shows the relaxation patterns around a Fr impurity and the induced volume changes of the host lattice for both cluster sizes. The relaxation patterns are the results of lattice statics simulations starting from the $a b$ initio forces at the ideal positions and the volume changes were calculated using the Kanzaki model described in Sec. III. It is seen, that the relaxation patterns around the impurity are practically the same, so the cluster size $N=59$ is sufficiently large to estimate the lattice relaxations around the large $s p$ impurities. Also the hyperfine fields at the impurity site do not differ between the two calculations, they are within $1 \mathrm{~T}$ from each other both for the ideal and the final relaxed positions.

The treatment of lattice relaxations within the KKR Green's-function formalism is somewhat complicated, because the Green's functions are represented as angular momentum expansions around the corresponding lattice sites, the host one around the unshifted (ideal) positions and the impurity one around the new shifted positions. While the reference Green's function for such shifted ("interstitial") positions can also be evaluated by a Brillouin-zone integration, we use here a simpler method introduced by Lodder, ${ }^{25}$ which is, however, limited to small displacements. In this scheme, the Green's function of the host crystal is transformed to the shifted positions by the so called $U$ transformation, which for small displacements couples an angular momentum quantum number $l$ with $l \pm 1$, so one has to increase the angular momentum cutoff of the radial functions, e.g., from $l_{\max }=3$ to $l_{\max }=4$, which is the reason to use as

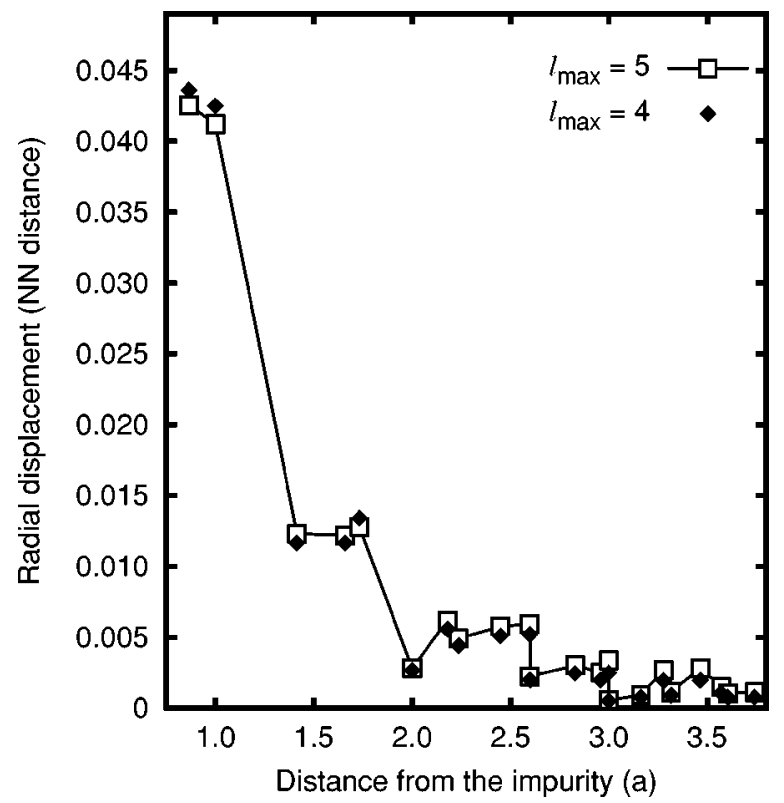

FIG. 3. Results of harmonic model simulations, i.e., $\Delta \Phi=0$ in the Kanzaki model, starting with the $a b$ initio forces from calculations, where the first and the second $\mathrm{NN}$ shells around a Fr impurity are displaced outwards $+5.0 \%$ and $+2.9 \%$ of the NN distance, respectively. Shown are the radial displacement patterns obtained using the $l$ cutoffs $l_{\max }=4$ and $l_{\max }=5$ in the $U$ transformation (see text).

high as $l_{\max }=4$ cutoff in this work. To test the quality of the $U$ transformation, a calculation for a $\mathrm{Fr}$ impurity in $\mathrm{Fe}$ was also done with the so-called void method, ${ }^{26}$ where the $U$ transformation is done with a higher $l$ cutoff than the selfconsistent parts of the calculation. Figure 3 shows the calculated lattice relaxation patterns around a Fr impurity obtained using the $l$ cutoffs $l_{\max }=4$ and $l_{\max }=5$ for the transformation and a cutoff $l_{\max }=4$ for the self-consistent parts. Both cases refer to calculations, where the first $\mathrm{NN}$ and the second $\mathrm{NN}$ shells were shifted radially outwards $5.0 \%$ and $2.9 \%$ of the $\mathrm{NN}$ distance, respectively, and the resulting $a b$ initio forces were used as input to the lattice statics simulations. It is seen, that the $U$ transformation with $l_{\max }=4$ is sufficiently accurate for moderate relaxations, say, less than $10 \%$ of the $\mathrm{NN}$ distance, which was also noted by Settels. ${ }^{26}$

To allow lattice relaxations, $20 \%$ smaller than touching MT spheres are used for the iron sites in all impurity calculations. For the impurity sites a touching MT radius is used since these atoms are not moved. The Green's function and the potential of the host crystal are recalculated for the $20 \%$ smaller MT sphere radius and the semicore contour discussed in the last subsection is used. It was checked for each impurity that the core charge density (without the semicore charge) was small enough at the sphere boundary, so that the treatment of the core electrons of the large impurity atoms was adequate within the relatively small MT sphere of host crystal.

\section{IMPURITY INDUCED LATTICE RELAXATIONS} IN bec Fe

In order to accelerate the determination of the equilibrium atomic positions from the $a b$ initio forces, lattice statics 


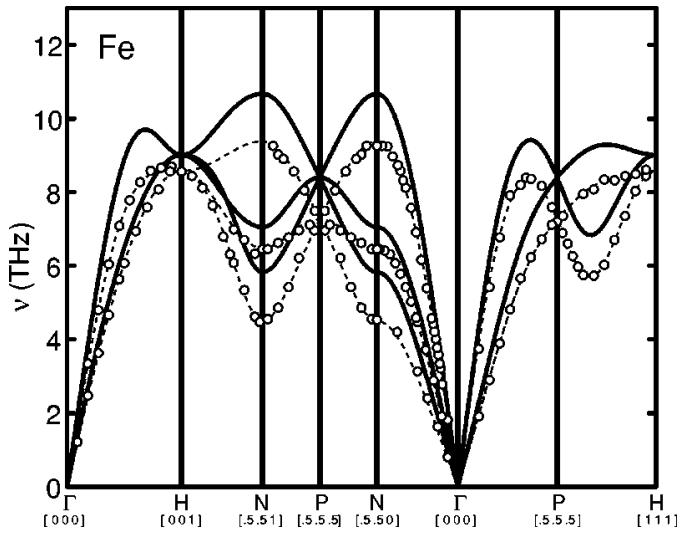

FIG. 4. Calculated phonon spectrum of bcc iron (solid lines). The experimental points $(\bigcirc)$ are from Ref. 28 and the thin dashed lines are a Born-von Karman fit to the experimental data.

method as described in Ref. 2 based on the Kanzaki method $^{27}$ is used. In the harmonic approximation the displacement pattern $\mathbf{s}^{n^{\prime}}$ is related to the force distribution $\mathbf{F}^{n}$ by $\mathbf{F}^{n}=\Sigma_{n^{\prime}} \Phi^{n n^{\prime}} \mathbf{s}^{n^{\prime}}$, where $\Phi$ denotes the coupling-constant matrix of the defect system. By splitting this up into the coupling-constant matrix $\Phi_{0}$ of the ideal crystal and the changes $\Delta \Phi$ induced by the defect, $\Phi=\Phi_{0}+\Delta \Phi$, the Kanzaki forces are defined by $\mathbf{F}_{K}^{n}=\Sigma_{n^{\prime}} \Phi_{0}^{n n^{\prime}} \mathbf{s}^{n^{\prime}}=\mathbf{F}^{n}$ $-\sum_{n^{\prime}}(\Delta \Phi)^{n n^{\prime}} \mathbf{s}^{n^{\prime}}$. In the calculations the changes of the coupling constants are restricted to the first two NN shells around the defect. The force pattern $\mathbf{F}_{K}^{n}$ is then applied on a hypothetical ideal lattice described by the calculated coupling constants $\Phi_{0}$ of the host and the displacements of all atoms are determined iteratively. The resulting positions $\mathbf{s}^{n}$ are then used in the next $a b$ initio study and the whole procedure is repeated until the displacements are converged. Only the first two NN shells are allowed to relax in the $a b$ initio calculations and the rest of the atoms are kept at the ideal positions to improve the embedding of the impurity cluster into the host crystal. In the densely packed bcc lattice of iron, only these two NN shells have significant relaxations, the forces on further atoms are small and they relax only a little (see Figs. 2, 3, and 6).

The ideal crystal coupling constants were determined by using a cluster of 59 perturbed iron potentials where the central atom was shifted along the [001] direction by a small amount $(0.5 \%$ of the lattice constant). Force constants up to five NN couplings were calculated from the $a b$ initio forces and from these the dynamical matrix and the phonon spectrum were constructed. The calculated phonon spectrum (solid line) and the experimental neutron-scattering data ${ }^{28}$ (open circles) are shown in Fig. 4. The thin dashed line is a Born-von Karman fit to the experimental points. The calculated phonon frequencies are a little bit too high but this is expected since the LSDA gives a lattice constant that is somewhat smaller than the experimental one and it also overestimates the bulk modulus. These are known to be the typical drawbacks of the L(S)DA when one considers the cohesive properties of the transition metals. Also the bump along the line $\Gamma-X$ arising from the second $\mathrm{NN}$ interactions seems to be an artifact of the LSDA calculation.

Figure 5 shows the calculated relaxation of the first and

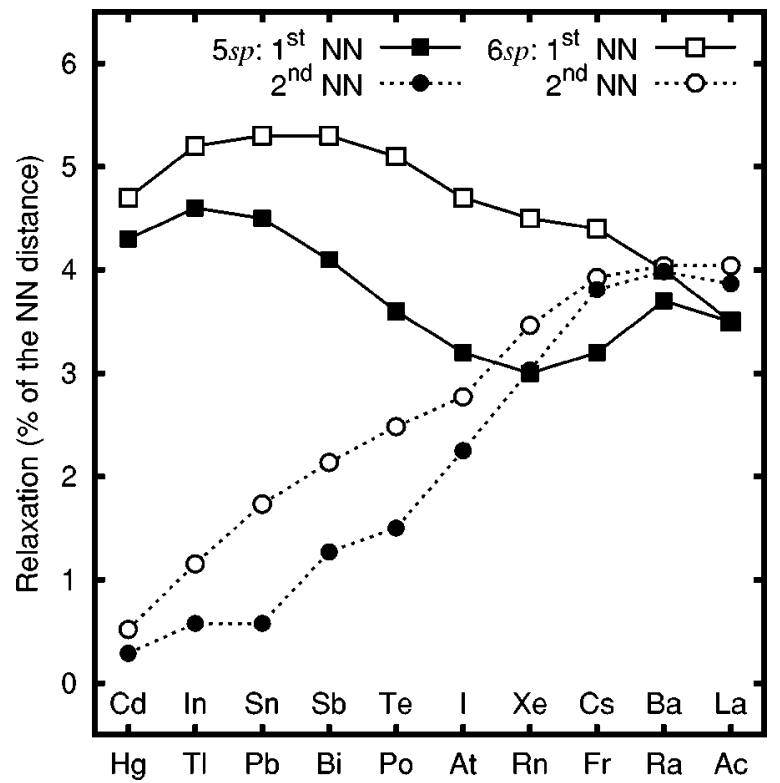

FIG. 5. Calculated relaxation of the first (squares) and the second (circles) $\mathrm{NN}$ shells around $5 s p$ and $6 s p$ impurities in iron.

second NN bcc iron shells around substitutional $5 s p$ and $6 s p$ impurities. For the $5 s p$ impurities the first $\mathrm{NN}$ atoms relax outwards $3.0-4.5 \%$ of the NN distance whereas for the larger $6 s p$ atoms the relaxations are somewhat larger (3.5$5.3 \%$ ). Surprisingly, the NN relaxations are largest for the tri- and tetravalent impurities and become somewhat smaller for the impurities at the end of the series. The relaxations of the second nearest neighbors increase about linearly with the valence and are at the end of the $s p$ series, i.e., at the beginning of the next row in the periodic table, even larger than the relaxations of the first neighbors. In this valence region the impurities of the $5 s p$ and $6 s p$ series are seen to relax the neighboring atoms about the same amount.

The systematic trend for the relaxations of the first and second NN shells becomes clearer, when one considers the forces on atoms in different shells. Figure 6 shows the forces on the first three neighboring shells around the impurities, calculated at the ideal lattice positions. The third NN forces (triangles) are multiplied by a factor of 10 in the figure and are seen to be more than an order of magnitude smaller than the forces on the first (squares) and second (circles) NN atoms. The same is true for the longer ranged forces. The NN forces are largest for the tri- and tetravalent impurities and then reduce to about $50 \%$ at the end of the series. Contrary to this the forces on the second neighbors are very small at the beginning and very large at the end of the series. This behavior of the first and second $\mathrm{NN}$ forces is counterintuitive to usual size arguments, from which one would always expect the NN forces to dominate. The third and further NN relaxations are much smaller than the relaxations of the two NN shells. One gets some idea of these further reaching distortions by looking at Fig. 2, where lattice distortions around a Fr impurity are shown. The distortion is seen to go rather far away from the impurity and is seen to expand the lattice (displacements are outwards), whereas the calculated $a b$ initio forces are not always pointing outwards for every shell included in the impurity cluster. This is due to the Friedel oscillations occuring in the iron host, so that the charge per- 


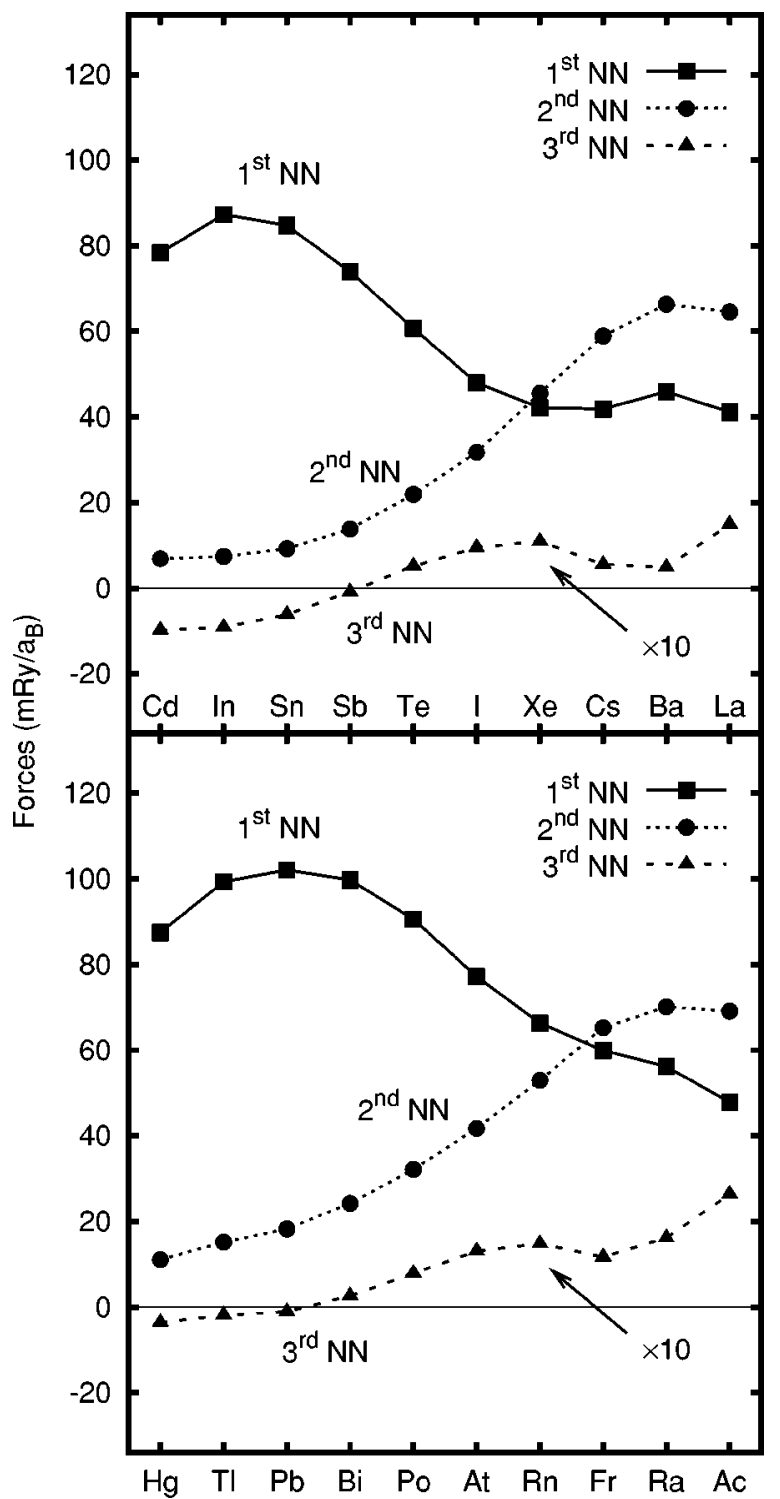

FIG. 6. The $a b$ initio forces on the first three NN shells around $5 s p$ and $6 s p$ impurities. The forces correspond to ideal lattice sites and the third $\mathrm{NN}$ forces are multiplied by 10 in the plot.

turbations have an decaying oscillatory character.

The Kanzaki forces $\mathbf{F}_{K}^{n}$ can be used to determine the volume change of the lattice $\Delta V$ induced by an impurity and it is given by ${ }^{27}$

$$
\Delta V=\frac{1}{3 K} \sum_{n} \mathbf{F}_{K}^{n} \cdot \mathbf{R}^{n},
$$

where $K$ is the bulk modulus of the ideal crystal and $\mathbf{R}^{n}$ are the lattice vectors. The largest contribution to the sum in Eq. (3) is coming from the first two NN shells around the impurity as can bee seen from Fig. 2, where the evolution of $\Delta V$ is plotted shell by shell together with the radial displacement fields for the clusters containing 59 and 169 perturbed potentials. It is seen that the first two NN shells make the main contribution to the volume change and the contribution of the further shells seems to be averaged out, so that the first two shells determine mainly the size of the volume change and the further shells seem to create some small oscillations

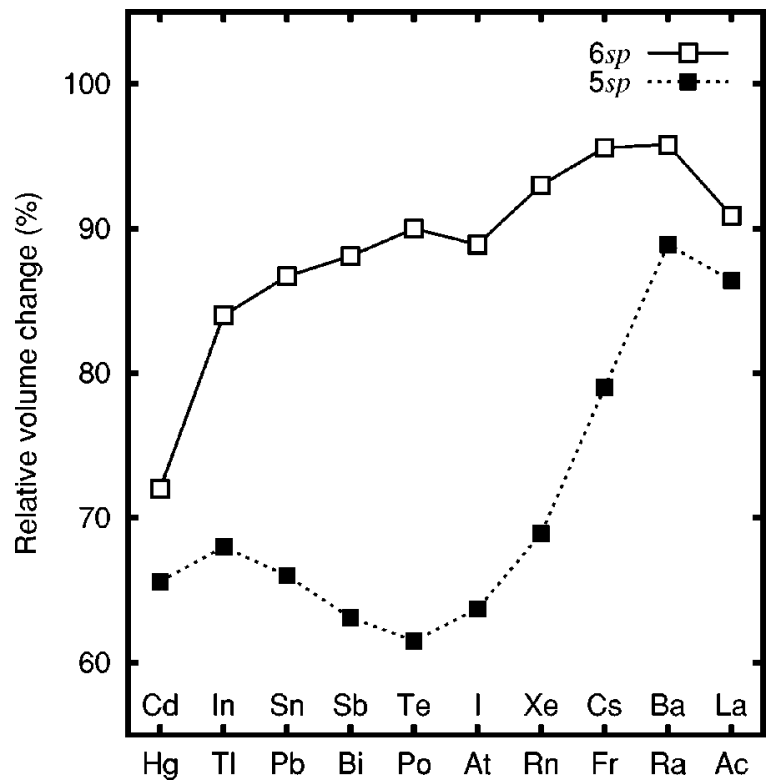

FIG. 7. Impurity induced volume changes in bcc Fe calculated using the Kanzaki model (3). Shown are the relative volume changes $\Delta V / V_{0}$ (percent of the elementary volume $V_{0}$ of $\mathrm{Fe}$ ) induced by the $5 s p(\boldsymbol{\square})$ and $6 s p(\square)$ impurities.

around this value. The calculated relative volume changes $\Delta V / V_{0}$ obtained from the Kanzaki model are presented in Fig. 7 ( $V_{0}$ is the elementary volume of Fe). These changes can also be experimentally obtained from lattice parameter measurements but for the most now studied defects the low solubility (if any) makes these measurements impossible. The volume changes follow the trend seen from the forces on the first and second neighbors, since the forces on the further away atoms are small. Therefore the curves for the volume changes show a two-peak structure, which is very pronounced for the $5 s p$ series but somewhat smeared out for the $6 s p$ series. The first peak is due to maximum of the $\mathrm{NN}$ forces at the beginning of the series, the second and larger peak obtains the largest contribution from the second NN forces being largest at the beginning of the next row. The largest volume changes are about $0.9 V_{0}$, which are really small compared to the huge atomic volumes of these impurities, which are, e.g., for Cs or Fr, up to 10-12 times larger than that of Fe. This behavior is very different from the one found for $3 d$ and $4 d$ impurities in $\mathrm{Cu}$, for which the volume changes scale reasonably well with the atomic volumes of the impurities introduced into the $\mathrm{Cu}$ host by substitution. In terms of elasticity language one could therefore classify the $s p$ impurities as highly compressible.

\section{HYPERFINE FIELDS OF IMPURITIES IN bcc Fe}

In the nonrelativistic theory the hyperfine field is determined by the Fermi contact interaction and given by

$$
H_{\mathrm{hf}}=\frac{8 \pi}{3} m(\mathbf{r}=0),
$$

where $m(\mathbf{r}=0)$ is the magnetization density at the nucleus. In this work the calculation of the hyperfine field is based on Breit's formula, ${ }^{29}$ which is the correct relativistic expression 
for hyperfine fields. We calculate the hyperfine fields by using the relativistic generalization ${ }^{30}$ of the contact term and neglect the orbital contribution in line with the scalar relativistic approximation used in our calculations. Since the considered $s p$ impurities have no genuine magnetic moments, the orbital contributions should be very small. Moreover the dipolar contributions vanish for the impurities due to the cubic symmetry of their surroundings. For the magnetic impurities, e.g., transition-metal atoms, the local magnetic moments give rise to large core polarization contributions to the hyperfine field, which, however, practically vanish for $s p$ impurities. Nevertheless, also here small core polarizations arise, which are included in the calculation. The major contribution for the "nonmagnetic" $s p$ impurities arises from the valence hyperfine field. Calculations have shown, that this field scales with the local $s$ moment of the impurities, ${ }^{30}$ which is induced by the hybridization of the impurity states with the spin dependent $d$ orbitals of the nearest neighbors. Thus the valence hyperfine field is basically caused by the population difference between the spin-up and spin-down valence $s$ states, but not by the spin polarization of the radial $s$-wave functions in the core region, which determines the hyperfine fields of the core electrons.

The calculated hyperfine fields of the $5 s p$ and $6 s p$ substitutional impurities in bcc Fe are compared with experiments and earlier KKR-ASA calculations in Fig. 8. Present full-potential KKR values are the solid lines with filled squares for the final relaxed positions and with solid circles for the ideal lattice positions. The KKR-ASA values taken from Ref. 12 are shown with dashed lines and the experimental ones ${ }^{8,9,13,14}$ with open triangles.

Let us first concentrate on the calculations performed without lattice relaxations. It is seen that both the fullpotential and the ASA calculations give almost identical hyperfine fields at the beginning of the series up to the rare-gas atoms. Contrary to this, at the beginning of each row in the periodic table the ASA trend differs considerably from the full-potential one. This is the critical region within each row, where the hyperfine fields change dramatically between neighboring elements and where therefore numerical details of the calculations could become very important. In particular for the alkali impurities the differences between ASA and full-potential results are unexpectingly large. Partly this might arise from the importance of nonspherical contributions to the potential. However, partly this could also arise from the improvements of the spherical potential parts, e.g., by including the full charge density in the evaluation of the spherical potential. A relevant improvement could also be the treatment of the semicore states as valence states, since for the alkali metals the semicore states are very shallow. It can be seen from Fig. 8 that the inclusion of lattice relaxations substantially improves the agreement with the experiment. This is particularly true for the $s p$ impurities in the first half of the series, where the hyperfine field is increased due to lattice relaxations. On the other hand, for the later $s p$ impurities the experimental hyperfine fields for the $s p$ impurities are not that well reproduced. In the $5 s p$ series the calculated hyperfine field of Cs is much too large. In the $6 s p$ series there are large discrepancies for At and Rn as well as for Ra and Ac. On the other hand, for the Fr impurity, the hyperfine field of which has only recently been measured,

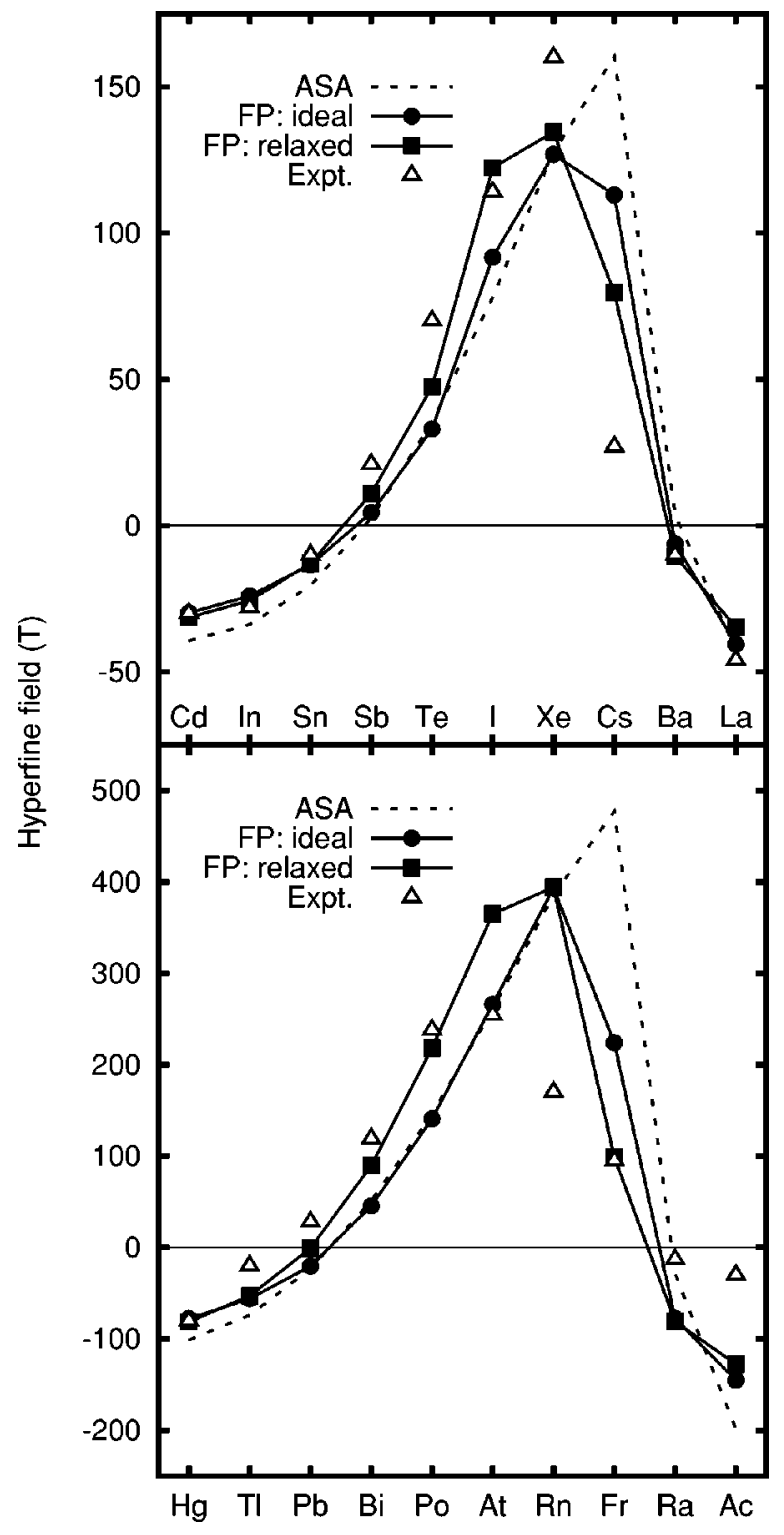

FIG. 8. Hyperfine fields of the $5 s p$ and $6 s p$ substitutional impurities in bcc Fe. Shown are the present full-potential values with (ם) and without (O) lattice relaxations and the ASA results (dashed line) of Akai et al. (Ref. 12). The experimental points $(\triangle$ ) are taken from Refs. 8, 9, 13, and 14.

the agreement between experiment and theory is excellent. However, in view of the errors for the neighboring element we have to consider this as a coincidence. Nevertheless, it is seen that the inclusion of lattice relaxations improves the agreement with the experiment, even if the relaxation effects are substantially smaller than we originally expected.

In order to search for possible errors in the theoretical treatment it is important to realize that the LSDA is not very good for bcc Fe. In the full-potential description the lattice constant is $3.6 \%$ smaller than the experimental one and also the calculated spin moment of $2.00 \mu_{B}$ is substantially smaller than the experimental one of $2.15 \mu_{B}$. On the other hand, it is known that the generalized gradient approximations (GGA) give a much better description of these properties, i.e., a nearly perfect lattice constant and magnetic moment. Actually, parallel to this work Cottenier and Haas ${ }^{31}$ calculated hyperfine fields of $4 d$ and $5 s p$ impurities in bcc 
iron by using a GGA. However, they used a very different method compared to the KKR Green's-function method used in this work, namely a full-potential linearized augmented plane-wave method (FLAPW) and they modeled the impurities by using a supercell geometry. The FLAPW+GGA results for the hyperfine fields of the $5 s p$ impurities compare well with the KKR+LSDA values (given in brackets), all values in tesla: $\mathrm{Cd}-44(-31), \mathrm{In}-35(-26), \mathrm{Sn}-15$ (-13), Sb 22 (11), Te 68 (48), I 129 (122), Xe 162 (135).

In order to simulate the lattice expansion caused by the GGA calculations using the LSDA and the experimental lattice constant of Fe were performed, leading to an $\mathrm{Fe}$ moment of $2.18 \mu_{B}$. This simulation is supported by recent KKR results $^{32}$ showing that for the same lattice constant the LSDA and the GGA yield the same moments, so that also similar hyperfine fields are expected. The hyperfine fields of the late $6 s p$ impurities Rn, Fr, Ra, and Ac did not change much with the lattice constant. Only the absolute values increased slightly, which is probably due to the increased host magnetic moment. Also the impurity induced forces did not change much and lattice statics simulations gave practically the same lattice distortions for the both lattice constant values. The above-mentioned results of Cottenier and Haas ${ }^{31}$ also support this observation. The hyperfine fields calculated with the FLAPW using the GGA for the $5 s p$ impurities are slightly larger than our results. They also did some calculations using both the LSDA and the GGA, but their results show that for the elements $\mathrm{Cd}-\mathrm{Xe}$ changes are small. The GGA is seen to increase the absolute values of the hyperfine fields slightly when compared to the LSDA ones calculated using the smaller LSDA lattice constant. Thus unfortunately we cannot pin down the origin for the large errors in the hyperfine fields of the late $6 s p$ impurities. We can only speculate that this might be an LSDA error or an error arising from the neglect of the spin-orbit coupling or maybe the experimental data are not sufficiently accurate.

\section{CONCLUSIONS}

We have performed first-principles full-potential calculations of the lattice relaxations and the hyperfine fields of heavy $s p$ impurities in iron. The treatment of these large impurities demands a correct treatment of the semicore elec- trons and it was shown how to implement the calculation of the semicore states in the KKR Green's-function formalism.

The calculated lattice relaxations around the $5 s p$ and $6 s p$ impurities were found to be relatively small, for the first and second NN shells less than $6 \%$ of the NN distance. The further relaxations were calculated using lattice statics methods and they were found to be much smaller, for the third NN shells a factor of 5 smaller than for the first two shells. We found the interesting trend that at the beginning of each $s p$ series the force on the $\mathrm{NN} \mathrm{Fe}$ atoms dominates, while at the end of the series the force of the second $\mathrm{NN}$ atoms are larger than the $\mathrm{NN}$ forces. We have also estimated the impurity induced volume changes of the lattice by using the Kanzaki method. The volume change induced by the $s p$ impurities was found to be around $60-95 \%$, surprisingly small in view of the king-size atomic volumes of the elements. Along the series the volume change exhibits a two-peak structure, resulting from the dominating contributions from the first and second NN shells.

The calculated hyperfine fields of $5 s p$ and $6 s p$ substitutional impurities reproduce the experimentally observed trend qualitatively well and also the quantitative agreement is good for the most of the impurities. The correct treatment of the semicore electrons of these large impurity atoms were found to be important and this probably explains the largest disagreement of the previous KKR-ASA calculations with experiments. In general the full-potential treatment improves the agreement with experiment and is, of course, mandatory for an accurate calculation of forces and relaxations. The inclusion of lattice relaxations in the calculations was also seen to improve the overall agreement of the calculated results with experiments, nevertheless leaving large discrepancies for $\mathrm{Xe}, \mathrm{Cs}$, At, and Rn impurities, the origin of which is not understood.

\section{ACKNOWLEDGMENTS}

This work was supported by a bilateral German-Greek cooperation grant and was partially funded by the TMR Network " $\mathrm{Ab}$ initio calculations of magnetic properties of surfaces, interfaces and multilayers" (Contract No. ERBFMRXCT960089).
${ }^{1}$ See, e.g., D. Guenzenberger and D. E. Ellis, Phys. Rev. B 49, 6004 (1994); K. Jackson, M. R. Pederson, and B. M. Klein, ibid. 43, 2364 (1991).

${ }^{2}$ N. Papanikolaou, R. Zeller, P. H. Dederichs, and N. Stefanou, Phys. Rev. B 55, 4157 (1997).

${ }^{3}$ N. Papanikolaou, R. Zeller, P. H. Dederichs, and N. Stefanou, Comput. Mater. Sci. 8, 131 (1997).

${ }^{4}$ U. Scheuer and B. Lengeler, Phys. Rev. B 44, 9883 (1991).

${ }^{5}$ H. W. King, J. Mater. Sci. 1, 79 (1966).

${ }^{6}$ W. B. Pearson, A Handbook of Lattice Spacings and Structures of Metals and Alloys (Pergamon, London, 1958), Vol. 1, p. 570; (Pergamon, Oxford, 1967), Vol. 2, p. 868.

${ }^{7}$ P. Herzog, Hyperfine Interact. 60, 563 (1990).

${ }^{8}$ C. J. Ashworth, P. Back, S. Ohya, N. J. Stone, and J. P. White,
Hyperfine Interact. 59, 461 (1990).

${ }^{9}$ B. Will, P. Herzog, R. Paulsen, J. Camps, P. De Moor, P. Schuurmans, N. Severijns, A. Van Geert, L. Vanneste, I. Berkes, M. De Jesus, M. Lindroos, and P. Van Duppen, Phys. Rev. B 57, 11527 (1998).

${ }^{10}$ H. Akai, M. Akai, S. Blügel, R. Zeller, and P. H. Dederichs, J. Magn. Magn. Mater. 45, 291 (1984).

${ }^{11}$ M. Akai, H. Akai, and J. Kanamori, J. Phys. Soc. Jpn. 54, 4246 (1985); 54, 4257 (1985); 56, 1064 (1987).

${ }^{12}$ H. Akai, M. Akai, S. Blügel, B. Drittler, H. Ebert, K. Terakura, R. Zeller, and P. H. Dederichs, Prog. Theor. Phys. Suppl. 101, 11 (1990).

${ }^{13}$ K. S. Krane, Hyperfine Interact. 15/16, 1069 (1983).

${ }^{14}$ G. N. Rao, Hyperfine Interact. 24-25, 1119 (1985). 
${ }^{15}$ H. Katayama-Yoshida, K. Terakura, and J. Kanamori, Solid State Commun. 29, 431 (1979).

${ }^{16}$ H. Katayama-Yoshida, K. Terakura, and J. Kanamori, J. Phys. Soc. Jpn. 46, 822 (1979); 48, 1504 (1980); 49, 972 (1980).

${ }^{17}$ J. Kanamori, H. Katayama-Yoshida, and K. Terakura, Hyperfine Interact. 8, 573 (1981); 9, 363 (1981).

${ }^{18}$ S. H. Vosko, L. Wilk, and M. Nusair, Can. J. Phys. 58, 1200 (1980).

${ }^{19}$ D. M. Ceperley and B. J. Alder, Phys. Rev. Lett. 45, 566 (1980).

${ }^{20}$ B. Drittler, M. Weinert, R. Zeller, and P. H. Dederichs, Solid State Commun. 79, 31 (1991).

${ }^{21}$ T. Korhonen, N. Papanikolaou, R. Zeller, and P. H. Dederichs, Philos. Mag. B 78, 429 (1998).

${ }^{22}$ K. Wildberger, P. Lang, R. Zeller, and P. H. Dederichs, Phys. Rev. B 52, 11502 (1995).

${ }^{23}$ Here we have used the same space division for the cluster as for the bulk, so the ideal crystal WS cells are used. One could also make a new Voronoi construction for the cluster, so one could use somewhat larger spheres, but the impurity semicore problem remains still for the very large impurity cores.
${ }^{24}$ Lloyd's formula is an extension of Friedel's sum rule to a perturbed cluster in a crystal. See P. Lloyd, Proc. Phys. Soc. London 90, 207 (1967); 90, 217 (1967). For its use within the KKR Green's-function method, see B. Drittler, M. Weinert, R. Zeller, and P. H. Dederichs, Phys. Rev. B 39, 930 (1989)

${ }^{25}$ A. Lodder, J. Phys. F: Met. Phys. 6, 1885 (1976).

${ }^{26}$ A. Settels, Ph.D. thesis, RWTH Aachen, 1999.

${ }^{27}$ G. Leibfried and N. Breuer, Point Defects in Metals I (Springer, Berlin, 1978).

${ }^{28}$ Metals: Phonon States, Electron States and Fermi Surfaces, edited by K.-H. Hellwege and J. L. Olsen, Landolt-Börnstein, New Series, Group III, Vol. 13, Pt. a (Springer-Verlag, Berlin, 1981), p. 180.

${ }^{29}$ G. Breit, Phys. Rev. 35, 1447 (1930).

${ }^{30}$ S. Blügel, H. Akai, R. Zeller, and P. H. Dederichs, Phys. Rev. B 35, 3271 (1987).

${ }^{31}$ S. Cottenier and H. Haas, following paper, Phys. Rev. B 62, 461 (2000).

${ }^{32}$ M. Asato, A. Settels, T. Hoshino, T. Asada, S. Blügel, R. Zeller, and P. H. Dederichs, Phys. Rev. B 60, 5202 (1999). 